\title{
Tangram: por que não se pode construir um quadrado utilizando exatamente 6 de suas peças?
}

\author{
José Antonio Novaes \\ Faculdade de Educação da Baixada Fluminense (FEBF) \\ novarsja@globo.com \\ Celso Marques da Silva Junior \\ Centro Federal de Educação Tecnológica Celso Suckow da Fonseca, Rio de Janeiro \\ celsomjr@ig.com.br
}

André Monteiro Novaes

Secretaria Municipal de Educação do Rio de Janeiro (SME-RJ)

andremnovaes@gmail.com

\begin{abstract}
Resumo
O Tangram é um milenar jogo chinês que pode ser utilizado como ferramenta pedagógica para abordagem de diversos temas no Ensino de Matemática como, por exemplo, frações, perímetros, áreas, simetrias e rotações. Neste contexto, um problema que ganha especial destaque é o da construção de quadrados utilizando as peças do Tangram, sem que haja sobreposição destas. Neste trabalho, serão exibidas as soluções possíveis, a menos de simetrias e rotações, quando se utilizam exatamente $1,2,3,4,5$ e 7 de suas peças e será dada especial atenção para a discussão sobre o porquê ser impossível a construção de um quadrado com exatamente 6 peças do Tangram.
\end{abstract}

Palavras-chave: Áreas. Álgebra. Jogos matemáticos. Radicais. Geometria.

\section{Tangram: Why is it not possible to build a square using exactly 6 of its pieces?}

\begin{abstract}
Tangram is an ancient Chinese game that can be used as a pedagogical tool to approach various issues in the Teaching of Mathematics, for example, fractions, perimeters, areas, symmetries and rotations. In this context, one problem that earns special mention is the construction of squares from pieces of a Tangram, without overlapping them. In this work, the possible solutions, unless symmetries and rotations, will be displayed when using exactly 1, 2, 3, 4, 5 and 7 pieces and, in particular, will be discussed why the impossibility of building square with exactly 6 of its pieces.
\end{abstract}

Keywords: Area. Algebra. Mathematical Games. Radicals. Geometry. 


\section{Introdução}

O Tangram é um milenar jogo chinês, cujo surgimento é desconhecido apesar de existirem diversas lendas para tal. Talvez, uma das mais comuns explicações para a sua origem seja a lenda do guerreiro que se apaixonou pela filha do Imperador e que, ao pedir a permissão para o namoro, recebeu o desafio de percorrer as fronteiras chinesas em sua totalidade e, ao regressar, representar tudo que havia visto ao Imperador utilizando somente as 7 peças de um espelho quadrado que havia se quebrado, o Tangram. Dessa forma, o Tangram é formado por 7 figuras geométricas planas - 5 triângulos, 1 paralelogramo e 1 quadrado - obtidos pela decomposição específica de um quadrado. $\mathrm{Na}$ verdade, como veremos na seção "Discussão do problema," na qual será apresentada em detalhes a construção do Tangram, os 5 triângulos em questão são semelhantes, havendo 2 pares de triângulos congruentes. Por este motivo, os 3 triângulos não congruentes são comumente chamados de triângulo grande, triângulo médio e triângulo pequeno. $O$ objetivo original do jogo é simplesmente a construção de figuras utilizando todas as 7 peças do Tangram, sem que haja sobreposição delas, mas o grande apelo geométrico do jogo nos permite torná-lo uma interessante ferramenta para o ensino de matemática.

Uma busca em bibliografias voltadas para o Ensino da Matemática (SANTOS; IMENES, 1987; MOURA, 1994; KALEFF, 1994, 2003; NOVAES, 2003) ou até mesmo na internet (EDUCAR, 2014; RACHACUCA, 2014; NPMAT, 2014) nos permite encontrar diversas curiosidades envolvendo Tangram, tais como desafios de construções a serem feitas utilizando todas as suas peças, conforme a regra original, ou utilizando apenas algumas das peças, numa variação da regra original, e até mesmo sugestões de atividades para explorar esta ferramenta em sala de aula. Em algumas destas referências, é possível encontrar a sugestão de construção de quadrados utilizando as peças do Tangram. Mais especificamente, são sugeridas construções de quadrados que utilizem 2, 3, 4, 5 ou 7 peças, sem que a possibilidade de construção com exatamente 6 peças seja abordada de forma tão frequente. Neste sentido, torna-se natural a pergunta:

“É possível construir um quadrado utilizando exatamente 6 peças de um Tangram?”

O objetivo deste artigo é discutir a questão apresentada acima, evidenciando que tal construção não é possível.

\section{Fundamentação teórica}

A utilização de materiais concretos e jogos em sala de aula é uma interessante opção para motivar questões e facilitar a compreensão de diversos conteúdos pelos alunos. Os Parâmetros Curriculares Nacionais são claros quanto a sua utilização e ao papel que cabe ao professor, de "[...]analisar e avaliar a potencialidade educativa dos diferentes jogos (BRASIL, 1997, p. 49)". 
Neste cenário, o professor sempre deve estar atento quanto à necessidade de complementar as discussões propostas em cima destes materiais com a formalização matemática, o que consiste em não apenas apresentar as devidas definições, como também em demonstrar formalmente as conjecturas geradas. Segundo Moura,

[...] os jogos são recursos importantes a serem utilizado pelo professor, desde que eles tenham clareza do seu papel e de que os jogos por si só não vão garantir a aprendizagem de certos saberes que precisam ser sistematizados e correlacionados à proposta e aos objetivos pedagógicos que se esperam atingir. (MOURA, 2003, p. 79-80)

Os jogos atraem a atenção e facilitam a concentração do aluno. Um aluno empenhado na resolução de um desafio produz muito mais que um aluno apático e desmotivado. O Tangram também pode ser utilizado para introduzir determinados conceitos como o de frações, perímetros, áreas, simetria, rotações, equivalência de figuras planas entre outros (NOVAES, 2003). No caso específico das áreas, é interessante notar que o professor pode, junto a seus alunos, determinar a área de todas as peças do Tangram utilizando apenas os axiomas de área. De fato, consideremos os axiomas abaixo descritos:

- Axioma A1: A toda região $\mathrm{S}$ do plano corresponde a um número maior que zero. A este número chamamos área da região e denota-se $\mathrm{A}(\mathrm{S})$.

- Axioma A2: Se uma região pode-se obter pela união de duas outras regiões, $\mathrm{S}=\mathrm{S} 1 \cup \mathrm{S} 2$ então $A(S)=A(S 1)+A(S 2)$. Este axioma pode ser generalizado para $n$ regiões.

- Axioma A3: Triângulos congruentes têm áreas iguais.

- Axioma A4: Se a região $\mathrm{S}$ é um retângulo de lados x e y então $\mathrm{A}(\mathrm{S})=\mathrm{x}$.y

Fixando, por exemplo, a área do triângulo pequeno como 1 u.a. (onde A1 é utilizado para tal) é fácil ver que as áreas das demais figuras do Tangram podem ser obtidas utilizando-se, apenas, os axiomas A2 e A3, já que as áreas do triângulo médio, paralelogramo e quadrado podem ser obtidas, pela aplicação do axioma A2, decompondo tais figuras em dois triângulos pequenos, bem como a área dos triângulos grandes equivalem a área de quatro triângulos pequenos.

Deste modo o Tangram pode, de forma lúdica, ser utilizado para apresentar conceitos abstratos de forma concreta. Como outro exemplo, um dos vários desafios do Tangram é entregar aos alunos as sete peças e solicitar que eles formem um quadrado com elas. Ora, algumas vezes nenhum aluno consegue executar esta tarefa de imediato. Neste caso, o professor pode intervir, criando desafios mais fáceis que indiquem o caminho aos alunos. Com o auxilio do professor, que deve ir aumentando a dificuldade dos desafios intermediários, os alunos devem conseguir concluir a tarefa proposta inicialmente, pois a intervenção do professor criará uma "zona de desenvolvimento proximal" como sugere Vygotsky (1998, p. 112): 
[...] ZDP é a distância entre o nível de desenvolvimento real, que se costuma determinar através da solução independente de problemas, e o nível de desenvolvimento potencial, determinado através da solução de problemas sob a orientação de um adulto ou em colaboração com companheiros mais capazes.

Além de ter a utilização educacional justificada acima, o Tangram pode ajudar a desenvolver algumas das inteligências múltiplas defendidas por Gardner. Por exemplo, a inteligência Viso Espacial, assim descrita,

[...] expressa a habilidade para pensar de maneira tridimensional (comprimento, largura e altura), além de perceber, criar e modificar imagens. Expressa a capacidade em produzir e entender informações gráficas, bem como a capacidade de perceber o mundo visual com precisão. É muito desenvolvida nas pessoas que têm grande facilidade de localização espacial e geográfica. (GARDNER ${ }^{1}, 1994$ apud ABRANTES, 2002)

Ao juntar peças, para tentar formar figuras, o aluno pode perceber que utilizando os dois triângulos pequenos pode formar um quadrado, um paralelogramo ou um triângulo médio que, em cada caso, estará identificando um par de lados congruentes dos triângulos pequenos na montagem da figura. Caso use lados não congruentes ele verá figuras de formas variadas, incluindo pentágonos, hexágonos, decágonos, entre outros. Obviamente, a inteligência Lógico Matemática, assim descrita,

[...] expressa a capacidade de calcular, quantificar, considerar proposições e hipóteses e realizar operações matemáticas, desde as mais simples, até as mais complexas. Expressa a sensibilidade e capacidade de discernir padrões lógicos ou numéricos, capacidade de lidar com longas cadeias de raciocínio e facilidade para o cálculo e para a percepção da geometria espacial. Diversos estudos mostram que, as pessoas que possuem esta inteligência muito desenvolvida, têm algumas características especificas. Normalmente são pontuais e não admitem atrasos, de qualquer espécie. (GARDNER,1994 apud ABRANTES, 2002)

Este tipo de inteligência também é contemplado na utilização com as atividades com Tangram. Como o aluno possivelmente fará alguns desenhos, a inteligência Pictórica também será desenvolvida. Caso o professor pretenda desenvolver a escrita para comparar linguagem Pictórica com língua materna, o Tangram estaria sendo usado para desenvolver a linguagem Verbolinguística. Há ainda a possibilidade de se fazer um trabalho em grupos e assim desenvolver-se-ia a inteligência social.

Posto todas as argumentações teóricas acima, passamos ao trabalho com o Tangram.

\section{Discussão do problema}

Inicialmente, visando àqueles menos familiarizados com o Tangram, apresentamos sua construção em detalhes:

\footnotetext{
${ }^{1}$ GARDNER, H. Estruturas da mente: A teoria das inteligências múltiplas. Porto Alegre: ARTMED, 1994.
} 
Figura 1 - Construção do Tangram

Construa um quadrado ABCD.

* Trace a diagonal AC.

* Trace o segmento JN, paralelo à diagonal $\mathrm{AC}$, onde $\mathrm{J}$ e $\mathrm{N}$ são os pontos médios dos segmentos $\mathrm{AD}$ e $\mathrm{DC}$, respectivamente.

* Trace o segmento BM, onde M é ponto médio de JN.

* Trace o segmento perpendicular a AC cuja uma extremidade é $\mathrm{N}$ e a outra está sobre AC.

* Trace o segmento paralelo a AD com

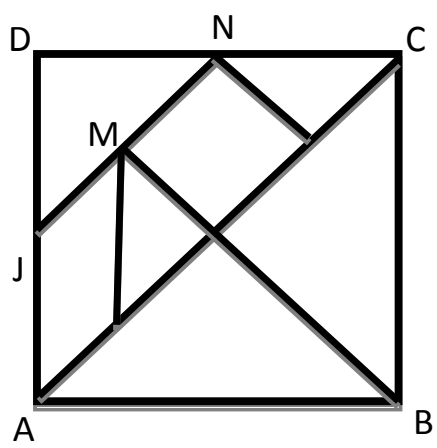
uma extremidade em $\mathrm{M}$ e outra sobre a diagonal AC.

O Tangram apresentado acima é o mais conhecido e comumente utilizado e, neste trabalho, iremos nos ater apenas a discussões envolvendo apenas este quebra-cabeça. Apesar disso, vale ressaltar que existem outros tipos de quebra-cabeças geométricos neste estilo, com quantidade e tipos de peças distintas (KALEFF, 2003; 2004), entre eles, o Tangram Quadrado de Quinze Peças Poligonais, o Tangram Quadrado de Quinze Peças com Formas Circulares, o Tangram de Lloyd, o Tangram Coração Partido, o Tangram Ovo Mágico, o Tangram Triangular e ainda o Tangram Pitagórico utilizado em uma das possíveis demonstrações do Teorema de Pitágoras.

A partir deste momento, dado um Tangram obtido como na Figura 1, utilizaremos, por simplicidade, como unidade de comprimento (1 u.c.) a metade da hipotenusa do triângulo pequeno. Neste caso, as sete peças do Tangram tem seus lados e suas áreas mensurados da seguinte forma:

Tabela 1 - Medidas de lados e áreas das peças do Tangram que são obtidas a partir de medida de comprimento fixada.

\begin{tabular}{|c|c|c|}
\hline Peças & Figura / Lados & Área \\
\hline Triângulos Pequenos & & $\mathrm{A}=1$ \\
\hline Triangulo Médio & & $\mathrm{A}=2$ \\
\hline
\end{tabular}




\begin{tabular}{|c|c|c|}
\hline Triângulos Grandes & A & \\
\hline Paralelogramo & & $\mathrm{A}=4$ \\
\hline Quadrado & & $\mathrm{A}=2$ \\
\hline & & \\
\hline
\end{tabular}

É evidente que não se perde generalidade fixando a metade da hipotenusa do triângulo pequeno como unidade de comprimento, como feito anteriormente, visto que qualquer outra escolha apenas tornaria o comprimento de cada lado das peças do Tangram um múltiplo do caso adotado anteriormente, ou seja, as peças semelhantes às obtidas pela escolha inicial. Além disso, como podemos observar através da Tabela 1, a partir da unidade de medida adotada obtém-se que todas as medidas de comprimento dos lados das peças do Tangram podem ser escritas na forma $a+b \sqrt{2}$, em que $a$ e $b$ são números inteiros. Evidentemente, a soma de quaisquer elementos desta forma ainda resultará em um elemento desta forma, visto que dados a, b, c e d inteiros, temos:

$$
(a+b \sqrt{2})+(c+d \sqrt{2})=(a+c)+(b+d) \sqrt{2} .
$$

Isto nos garante que qualquer quadrado formado por peças do Tangram terá a medida de seu lado escrita na forma $a+b \sqrt{2}$, com a e $\mathrm{b}$ inteiros. De fato, qualquer quadrado formado a partir do Tangram terá seu lado medindo o mesmo valor de algum dos lados das peças ou uma soma ou subtrações de medidas de lados de peças do Tangram. 
Por outro lado, voltando à questão apresentada inicialmente neste artigo, apresentamos na Tabela 2 todas as soluções, a menos de procedimentos relacionados às simetrias e rotações, para o problema da construção de um quadrado utilizando exatamente 2, 3, 4, 5 ou 7 peças de um Tangram, sem que haja sobreposição.

Tabela 2 - Soluções para o problema da construção de um quadrado utilizando exatamente $2,3,4,5$ ou 7 peças de um Tangram.

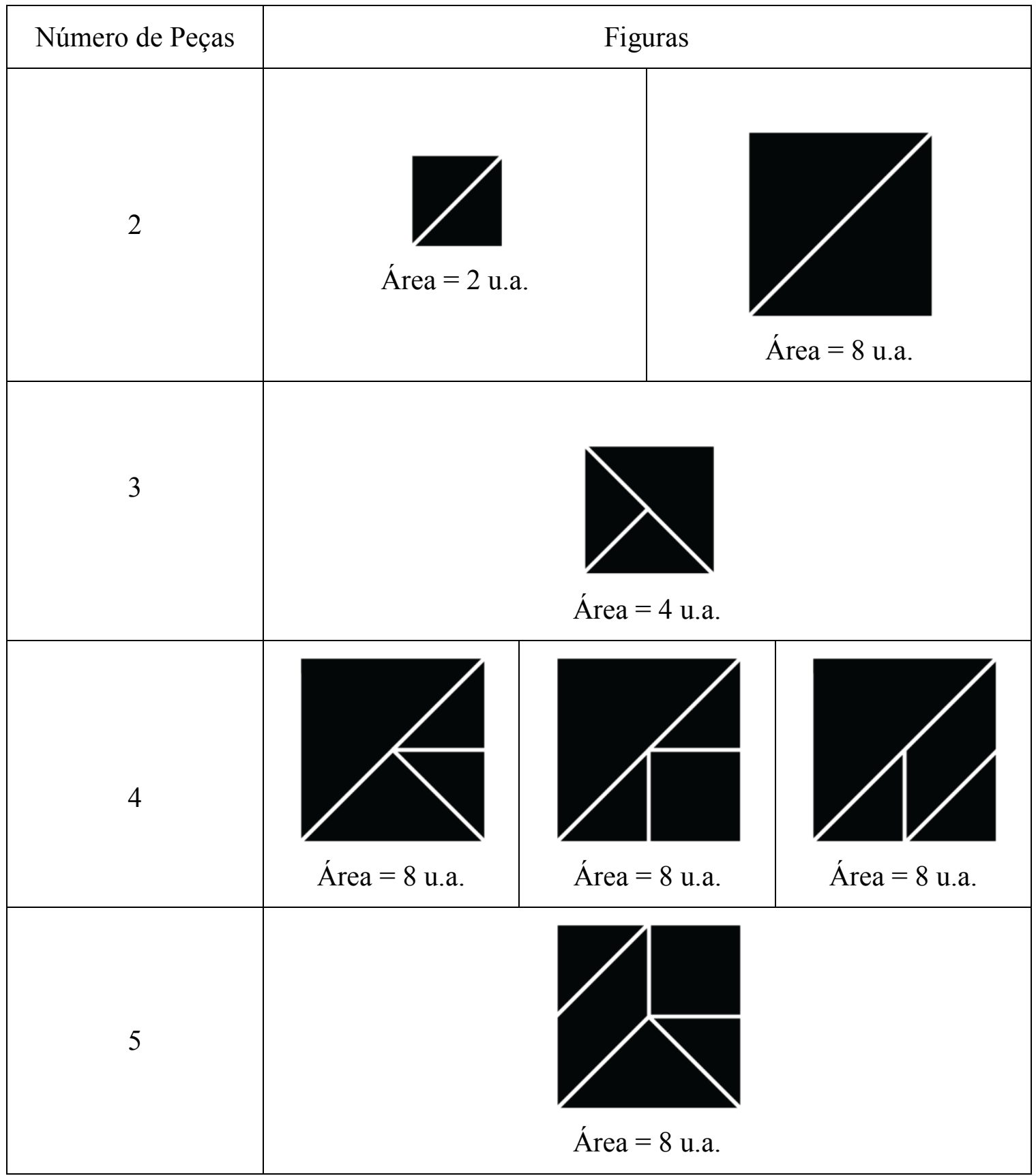




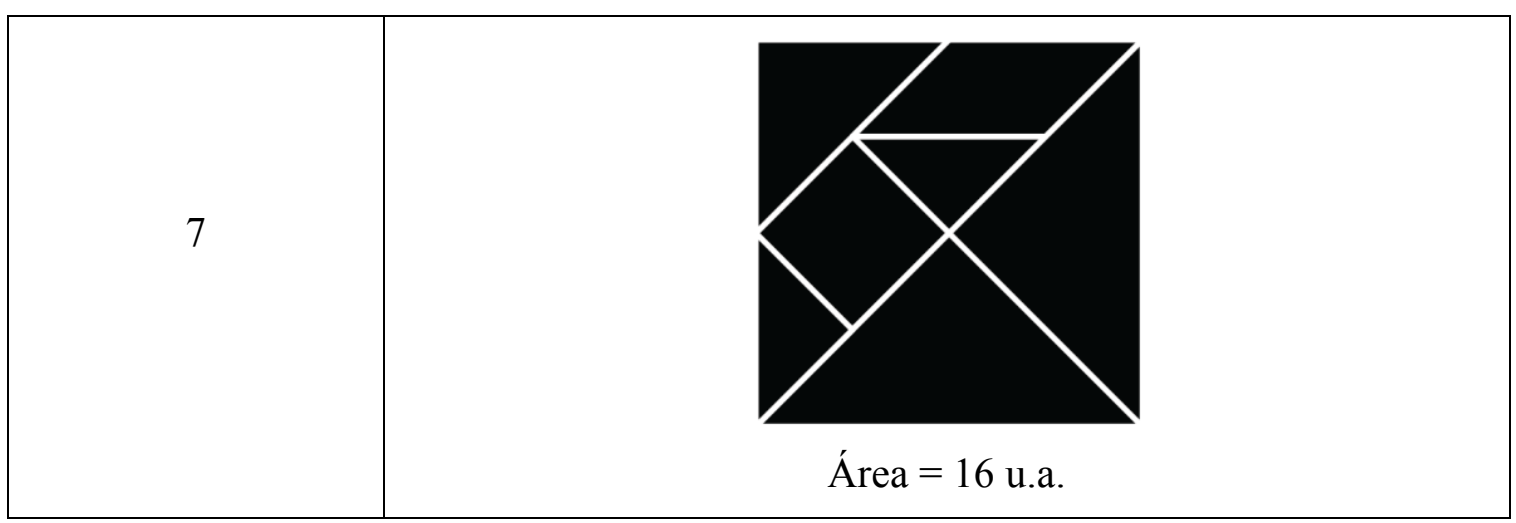

Já para o problema da construção de um quadrado utilizando exatamente 6 peças do Tangram, apresentamos abaixo as possíveis áreas que seriam obtidas, caso tal construção fosse possível:

- Área medindo 15 u.a. - Obtida pela retirada de um triângulo pequeno.

- Área medindo 14 u.a. - Obtida pela retirada de um quadrado ou um paralelogramo ou triângulo médio.

- Área medindo 12 u.a. - Obtida pela retirada de um triângulo grande .

Sendo assim, lembrando que a medida do lado de um quadrado é igual à raiz quadrada de sua área, a construção de quadrados utilizando exatamente seis peças do Tangram, se possível, resultaria em quadrados cujos lados mediriam, respectivamente, $\sqrt{15}$ u. c., $\sqrt{14}$ u. c. ou $\sqrt{12}$ u. c.. Mas estes valores não podem ser escritos na forma $a+b \sqrt{2}$ com a e $b$ números inteiros e, como visto anteriormente, a partir da unidade de comprimento fixada, qualquer quadrado formado por peças do Tangram tem a medida de seu lado escrita nessa forma. Logo, supondo a construção em questão possível chegamos a um absurdo e, portanto, conclui-se não ser possível construir um quadrado utilizando exatamente 6 peças de um Tangram, sem que haja sobreposição.

Expandindo os resultados acima encontrados à proposta apresentada em Gênova (1990, p. 28) de utilização de dois Tangrans para criar outras figuras, poderíamos questionar a possibilidade da construção de quadrados utilizando mais de 7 peças. Neste caso, identificam-se, por exemplo, quadrados com 8 peças (obtidos utilizando-se um Tangram completo acrescido de uma das figuras de área 2, isto é, um quadrado, triângulo médio ou paralelogramo) e ainda com 14 peças, nos quais os dois Tangrans completos são utilizados. No primeiro caso, o quadrado resultante tem área medindo 18 u.a.e lado $3 \sqrt{2}$ u. c. e, no segundo caso, mede 32 u.a. e lado $4 \sqrt{2}$ u. c.. 
Figura 2 - Quadrados formados utilizando 8 peças (dois Tangrans)
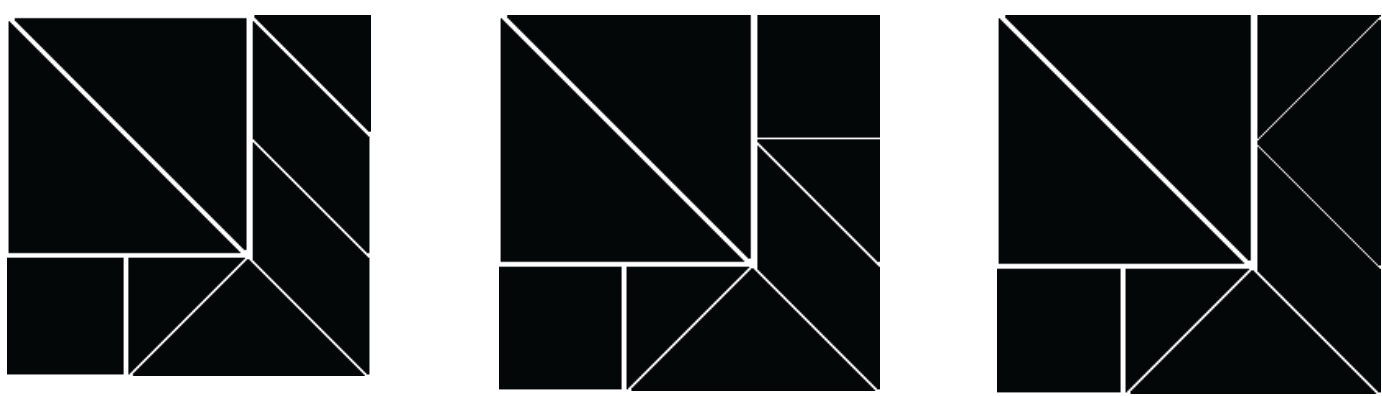

Figura 3 - Quadrado formado utilizando 14 peças (dois Tangrans).

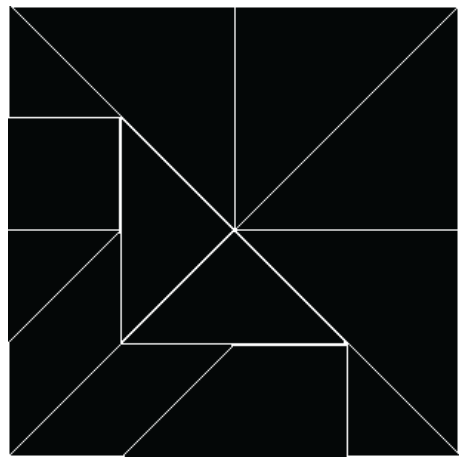

\section{Outra visão do problema}

É interessante notar que alternativamente a explicação apresentada, na última seção, para o fato de não ser possível construir um quadrado utilizando exatamente 6 peças do Tangram, poderia ser utilizado o conceito de Corpo (GONÇALVES, 2007). De fato, é amplamente conhecido que o conjunto $Q[\sqrt{2}]=\{a+b \sqrt{2}: a, b \in \sqrt{2}\}$ é um corpo. Como visto anteriormente, diante da unidade de comprimento escolhida, se o lado de uma peça do Tangram mede $x$ u.c então $x \in Q[\sqrt{2}$ ]. Mais ainda, como $Q[\sqrt{2}]$ é um corpo, é fechado para somas e diferenças e, portanto, o comprimento do lado de qualquer quadrado formado com peças desse Tangram também será um elemento de $Q[\sqrt{2}]$. Por outro lado, como também já mencionado na seção anterior, se fosse possível construir um quadrado utilizando exatamente 6 peças do Tangram ele deveria ter o lado medindo $\sqrt{15}$ u.c., $\sqrt{14}$ u.c. ou $\sqrt{12}$ u. c.. Como $\sqrt{15}, \sqrt{14}, \sqrt{12} \notin \mathrm{Q}[\sqrt{2}]$, verificamos ser impossível a construção desejada. 


\section{Considerações finais}

O Tangram é um material cada vez mais presente no Ensino da Matemática. Dentre as diversas atividades possíveis para sua utilização, temos a construção de quadrados utilizando suas peças. Aqui, os autores destacam mais uma vez que, apesar de ser conhecido o fato de ser impossível a construção de um quadrado utilizando exatamente 6 peças, não há uma ampla discussão do porquê tal construção não ser possível. A demonstração deste fato, exibida neste artigo, pode ser levada para dentro de sala de aula, até mesmo para alunos do Ensino Básico. Mais ainda, tal discussão pode ser utilizada como exemplo para mostrar aos alunos que, embora a utilização do material concreto seja ótimo para facilitar a compreensão de conceitos e até mesmo gerar conjecturas, sua utilização sempre deve vir acompanhada de embasamento teórico. Basta que o professor permita que os alunos observem que, mesmo após muitas tentativas da construção do quadrado utilizando exatamente 6 peças do Tangram, sempre seria possível que continuassem se perguntando: "Será que não existe mesmo uma forma, ainda não pensada por nós, de fazer tal construção?" A formalização da demonstração vem responder a este questionamento, de forma negativa.

Por fim, destacamos o fato de que mesmo um "problema simples" como o da construção de quadrados a partir do Tangram pode ser utilizado para gerar um interessante exemplo em um curso introdutório de Álgebra, em particular, na discussão de conjuntos fechados para a operação de soma, o que pode preceder a introdução dos conceitos de Anéis e Corpos, por exemplo. Na verdade, a utilização de materiais concretos e jogos, de forma geral, ainda está pouco presente no ensino universitário brasileiro, apesar dos diversos artigos apontando para as contribuições do lúdico no processo de ensino e aprendizagem em Matemática.

\section{Referências}

ABRANTES, J. A Ergonomia Cognitiva e as Inteligências Múltiplas. Anais VIII Simpósio de Excelência em Gestão e Tecnologia. Rio de Janeiro: UERJ, 2012. Disponível em http://www.aedb.br/seget/artigos11/55314676.pdf.

BARBOSA, João Lucas Marques. Geometria Euclidiana Plana, Coleção do Professor de Matemática. Rio de Janeiro: SBM, 2000.

BRASIL. Ministério da Educação. Secretaria de Educação Fundamental. Parâmetros Curriculares Nacionais: Matemática ( $1^{\circ}$ e $2^{\circ}$ ciclos do ensino fundamental). v. 3. Brasília: MEC, 1997.

BRASIL. Ministério da Educação. Secretaria de Educação Fundamental. Parâmetros Curriculares Nacionais: Matemática. ( $3^{\circ}$ e $4^{\circ}$ ciclos do ensino fundamental). Brasília: MEC, 1998.

EDUCAR / <http://www.espacoeducar-liza.blogspot.com.br/2011/07/atividades-com-oTangram.html $>$. Acesso em 21 de abril de 2014.

ELFFERS, J. Tangram: The ancient chinese shapes game. Londres: Penguim Books, 1976 GÊNOVA, A. C. Brincando com Tangram em Origami, São Paulo: Global Editora e Distribuidora Ltda, 1990 
GONÇALVES, Adilson. Introdução à Álgebra, Projeto Euclides, Rio de Janeiro: IMPA, 2001.

KALEFF, A. M. M. R. Tangrans geométricos especiais.

$<$ http://www.uff.br/cdme/tangrans_geometricos/index.html $>$. Acessado em 22 de abril de 2014.

KALEFF, A M. M. R.; HENRIQUES, A.; REI, D. M.; FIGUEIREDO, L. G. Desenvolvimento do pensamento geométrico: Modelo de Van Hiele, Bolema, v.10. 21-30, 1994

KALEFF, A. M. M. R., REI, D.M., e GARCIA, S. S. Quebra-cabeças geométricos e formas planas. $3^{\mathrm{a}}$ ed. Niterói: EdUFF, 2003

MACHADO, N. J. Matemática e educação: alegorias, tecnologias e temas afins. $2^{\text {a }}$.ed., São Paulo: Cortez, 1995

MOURA, M. C. O desafio de articular as estratégias com o desenvolvimento de competências gerenciais: um estudo de caso. São Leopoldo: Unisinos, 2003 (Dissertação de Mestrado).

MOURA, M. O. A Séria Busca do Jogo: do Lúdico na Matemática. Educação Matemática em Revista. Sociedade Brasileira de Matemática, 1994

NOVAES, J. A. Desafios, Frações, Áreas e Expressões Algébricas com Tangram, Anais $3^{\circ}$

EEMAT - RJ: Rio de Janeiro 2003, (CD-ROM).

NPMAT / <http://www.pt.slideshare.net/NPMat/atividade-com-o-Tangram-6093926>. Acesso em 21 de abril de 2014.

PROJETO FUNDÃO, UFRJ. Coordenadoras: Maria Laura M. L. Lopes e Lilian Nasser Geometria na era da imagem e do Movimento, Rio de Janeiro: Editora UFRJ, 1996.

RACHACUCA / <http://www.rachacuca.com.br/jogos/Tangram>. Acesso em 30 de abril de 2014.

SANTOS, C.H.; IMENES, L.M.P. Tangran: Um Antigo Jogo Chinês nas Aulas de Matemática. Revista de Ensino de Geometria, 1987.

VYGOTSKY, Lev Semenovich. A formação social da mente. $6^{\mathrm{a}}$ ed, São Paulo: Martins Fontes, 1998.

Submetido em maio de 2014 Aprovado em novembro de 2014 